\title{
Application of Axiomatic Design principles in conceptual design
}

\author{
Josipa Delaš ${ }^{1}$, Stanko Škec ${ }^{1, *}$, and Mario Štorga ${ }^{1}$ \\ ${ }^{1}$ Faculty of Mechanical Engineering and Naval Architecture, University of Zagreb, 10000 Zagreb, Croatia
}

\begin{abstract}
The main objective of this paper is to propose a modified methodology for concept evaluation by applying Axiomatic Design principles. Several drawbacks were recognised during the literature review and application of established Axiomatic Design principles that limit its use for concept evaluation. These drawbacks include the lack of analysis of concepts that violate the Independence Axiom, the application to concepts that are not generated with Axiomatic Design and inclusion of constraints and requirements in the evaluation process. The proposed methodology consists of four steps of which the first one is to analyse the compliance of concepts with a set of functional requirements. Afterwards, to determine the possible violation of the Independence Axiom, non-diagonal elements need to be examined and reangularity and semiangularity values calculated for each concept. Finally, concepts are evaluated in terms of Information Axiom to include requirements, criteria and constraints other than functional requirements. Applying Information Axiom to all concepts regardless of Independence Axiom violation provides insight into the complexity of concepts. The proposed methodology was applied to mobility scooter conceptual design conducted in cooperation with an industrial partner. The partner company provided input and system constraints at the beginning of the project and guidelines for concept development. Constraints were taken into consideration by applying the Information Axiom in which constraints are compared with values measured on prototypes.
\end{abstract}

\section{Introduction}

A concept is an approximate description of the technology, working principles, and form of the product [1]. In a sufficiently developed concept, its aspects (e.g. ease of use, aesthetic, functionality etc.) can be evaluated. Concept evaluation is used to determine which concepts have the highest potential of becoming a quality product [2]. The evaluation usually occurs at the end of the conceptual design phase when developing a new product or when selecting the best concept variant among existing ones [3]. The evaluation includes a comparison of concept variants or a comparison of a concept variant with the defined ideal solution [3]. Along with several existing methods and tools, Axiomatic Design (AD) theory can also be applied for concept evaluation [4]. Application of Axiomatic Design principles usually defines how close the alternative is to the ideal one as opposed to directly comparing alternatives to each other. According to [5], ideal concepts in Axiomatic Design can satisfy the identified customer needs and are scarcely affected by sensitivity to possible alterations in later phases. Concepts' ideality is achieved by proper implementation of the Independence Axiom and, subsequently, the Information Axiom. Along with other AD principles (e.g. domains, mapping process, decomposition, hierarchy, and zigzagging [6]), axioms provide the systematic basis for solving design problems in various design areas.

One of the main advantages of Axiomatic Design, in terms of concept evaluation, is the timely detection of internal technical conflicts in generated concepts through the Independence Axiom [7]. Other advantages include the ability to evaluate complete and incomplete information criteria together [8] and visualise dependencies between design parameters [9]. Further on, the application of the Information Axiom can be used to calculate the designs' complexity [6].

When developing new concepts using AD principles, evaluation is an integral part of the design process. Axiomatic Design principles enable continuous evaluation of concepts that are being generated and improved since the conformance to axioms can be checked at every step. However, evaluation is often applied at the end of the conceptual design phase to concepts that were not solely generated using $\mathrm{AD}$ principles. Defining the best concept among them is time-consuming compared to other concept evaluation methods, such as concept scoring and Pugh's method [7]. This is due to the process of finding an independent set of functional requirements (FRs) and design parameters (DPs), which is iterative especially for complex designs.

This paper provides an analysis of existing principles for concept evaluation with Axiomatic Design and suggests certain improvements to evaluation methodology. These improvements are a part of the proposed modified concept evaluation methodology which aims to resolve drawbacks observed in literature as well as in the implementation of $\mathrm{AD}$ principles in concept evaluation. A case study serves as the validation of the proposed methodology which, combined with the conducted literature review, provided the basis for the discussion and the conclusion.

\footnotetext{
* Corresponding author: stanko.skec@fssb.hr
} 


\section{Theoretical background}

Design using $\mathrm{AD}$ principles begins with defining functional requirements by reformulating customer needs (CNs) in a way that completely characterises the functional needs of a product. This first step enables comparison of different concepts designed to satisfy the same customer needs. The process of defining or selecting a DP that satisfies a certain FR is called the physical mapping [10]. Definition of DPs on higher decomposition level as specific solutions should be avoided, due to the risk of constraining the definitions of lower-level FRs and DPs. Instead, they can represent conceptual entities whose definition is extracted from the corresponding FR to prevent making early choices. Designs are detailed by decomposing FRs and DPs from a high level of abstraction to lower levels which contain detailed modularity elements [9]. The decomposition is achieved by zigzagging from FRs and DPs on different levels. From DPs on the higher-level, we define FRs on the lower level that completely satisfy the highest-level FR. DPs are then defined for lower-level FRs accordingly. These relationships between FRs and DPs are recorded in the design matrix.

The design matrices present the basis for the concept evaluation in terms of Axiomatic Design principles. The relationship between a specific FR and a corresponding DP found in the design matrix is called coupling. The Independence Axiom says that in an acceptable design, FRs and DPs are related in such a way that a specific DP can be adjusted to satisfy its corresponding FR without affecting other FRs [11]. Designs defined as uncoupled have coupling only in the main matrix diagonal and completely fulfil the Independence Axiom. The decoupled design has couplings on one side of the main matrix diagonal as well and therefore partially satisfies the Independence Axiom. Coupled design violates the Independence Axiom as it has couplings on both sides of the main matrix diagonal.

Concept evaluation using AD principles is based on a claim that a good concept completely satisfies the Independence Axiom. Depending on the number of couplings and design matrix size, in certain cases, it can be easy to distinguish which concept satisfies the Independence Axiom the most (by only looking at the design matrices). In other cases, independence is measured by calculating reangularity (R) and semiangularity (S). Reangularity relates the angles between the axes of the design parameters, while semiangularity measures the magnitude of the diagonal elements [12]:

$$
\begin{aligned}
& R=\prod_{\substack{i=1, n-1 \\
j=1+i, n}} \sqrt{1-\frac{\left(\sum_{k=1}^{n} A_{k i} A_{k j}\right)^{2}}{\left(\sum_{k=1}^{n} A_{k i}^{2}\right)\left(\sum_{k=1}^{n} A_{k j}^{2}\right)}} \\
& S=\prod_{j=1}^{n} \frac{\left|A_{j j}\right|}{\sqrt{\sum_{k=1}^{n} A_{k j}^{2}}}
\end{aligned}
$$

In these equations, $A$ refers to the value inside the design matrix in the row defined with the first index and the column defined with the second index.

$R$ value decreases as the degree of coupling increases. For $R=S=1$, the design is completely uncoupled, and for $R=S<1$ the design is decoupled. This calculation presents the basis for the coupling analysis which determines whether the Independence Axiom has been satisfied, and is implemented as the first part of the evaluation using AD principles. Procedure found in [11] is the most common for conceptualisation and analysis of the generated concepts with Axiomatic Design. It states that if multiple designs satisfy the Independence Axiom, the best among them is chosen by utilising the Information Axiom [Fig. 1]. The Information Axiom states that the information content in a design should be minimised. In other words, the design that results in the highest probability of meeting design specifications is the best one [11]. The highest probability of success indicates the lowest amount of information needed to manufacture (produce) the design [10]. However, the Independence Axiom is often satisfied, and thereby the Information Axiom is not utilised as a part of the evaluation procedure [8].

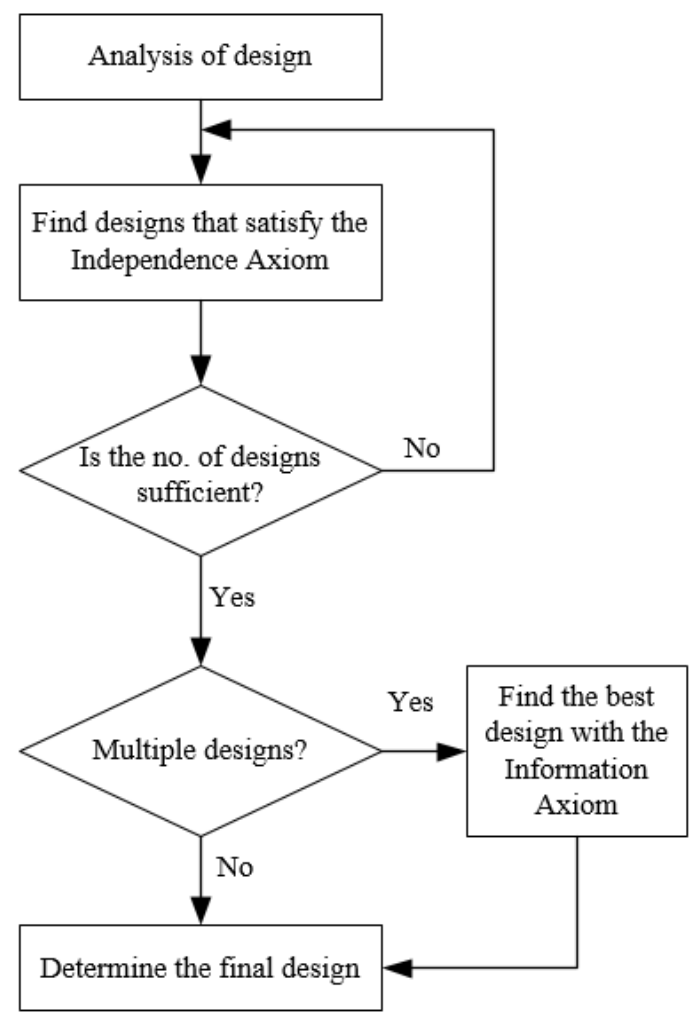

Fig. 1. Flowchart of the application of Axiomatic Design [11]

Several studies of evaluation with Axiomatic Design principles have been conducted ([13]-[15]) based on the aforementioned procedure. For example, AD principles were used to analyse and improve designs of emergency core cooling systems by complying to the Independence Axiom and, afterwards, the best among them was chosen with the Information Axiom [13]. Further, evaluation of the suspension systems was conducted by applying the 
axioms and analysing coupling to improve ride comfort [14]. In a case study that evaluated existing friction devices [15], the Information Axiom is applied even though none of the designs satisfies the Independence Axiom. In some studies, the evaluation was conducted as part of the application of Axiomatic Design principles for product development [16][17].

Based on these and other formerly conducted studies three drawbacks of concept evaluation with Axiomatic Design were recognised. To start with, problems occur when concepts that weren't generated with $\mathrm{AD}$ principles are being evaluated since the evaluation procedure itself doesn't alternate the relationships between defined FRs and DPs. To be more precise, procedure for evaluation is not defined if concepts that weren't generated using $\mathrm{AD}$ principles violate the Independence Axiom. However, concepts that are chosen for detailing can be subsequently modified by taking into consideration recognised couplings.

Secondly, there is a lack of guidelines for incorporating different types of requirements and criteria in concept evaluation. For instance, non-technical requirements are defined as constraints and, as such, are not part of the design matrix (and, consequently, not part of the evaluation) [7]. This exclusion of non-technical requirements can result in unaesthetic and costly products that fail to satisfy customer needs [11].

Thirdly, decomposition can include non-essential functions, which aim to increase attractive qualities of a product [18]. For instance, primary functions of a mobile phone are to send and receive phone calls, provide access to the Internet, take photos etc., but adding additional features to the camera can make the mobile phone more desirable. This approach leads to concepts that have a different set of FRs, even though they satisfy the same customer needs (CNs). When using $\mathrm{AD}$ principles for evaluation often certain concepts don't fulfil all identified FRs. As such, concepts have design matrices of various sizes what makes a comparison of concepts difficult. In other words, it is hard to achieve the ranking of alternatives since $\mathrm{AD}$ principles usually compare concepts to the ideal one.

Building on these premises, the following research methodology was used to structure and conduct this study.

\section{Research methodology}

The literature review was conducted during the preliminary research phase to analyse existing evaluation procedures based on Axiomatic Design principles.

Initial concept evaluation was done based on [11], which, along with the analysis of existing case studies, revealed its drawbacks. This procedure suggests applying Axiomatic Design in the same way for different purposes - concept development, analysis of existing designs and design improvement [11]. As such, it seems to be too generic and does not provide sufficient guidelines for the concept evaluation itself. For instance, in a case study of evaluating in-pipe robot design [16], dependencies of FRs and DPs were observed only at their own level of decomposition. This type of dependency analysis could lead to unrecognised couplings that, consequently, cause design issues in later stages of the design process. Other examples found in literature usually define a different design matrix for each concept. In such cases, concepts aren't compared to one another but only tested to see if and to what extent do they satisfy the Independence Axiom.

To address issues identified during the initial concept evaluation, the proposed methodology suggests creating a single design matrix equal for all developed concepts that includes their FRs and corresponding DPs. Mutual comparison of concepts can provide further insights into their strengths and weaknesses. Decomposition of a single concept rarely shows which FRs are missing, whereas having multiple concepts defined with the same FRs can indicate which FRs haven't been fulfilled by different concepts. Therefore, the analysis of fulfilment of FRs is set as a first step of the concept evaluation with the modified procedure. The second step, the analysis of non-diagonal elements, is added for similar reasons. An indication of mutual couplings can also help the designer to recognise reoccurrence of specific design problems, and imply potential solutions through comparison with other matrices. Examples found in the literature mostly aim at an analysis of concepts that are being generated by considering the Independence Axiom. Concepts that were not generated with AD principles often cannot be altered in such way, and therefore this methodology proposes an additional application of the Information Axiom to better inform a designer before deciding on a potential concept that should be further developed.

In order to illustrate the application of the modified methodology for concept evaluation, it was applied to mobility scooter conceptual design conducted in cooperation with an industrial partner.

\section{Proposed modified methodology for concept evaluation}

To tackle the previously mentioned problems in Sections 2 and 3, this paper proposes the modified concept evaluation methodology [Fig. 2]. The proposed procedure is primarily intended for evaluation of concepts that weren't generated with AD principles. In such cases, FRs are set after working principles of a product have already been defined. Such concepts cannot be changed to satisfy the Independence Axiom since alterations aren't a part of the evaluation process.

Unlike the procedure provided in [Fig. 1] where evaluation begins with the Independence Axiom, in modified methodology presented in this paper evaluation starts with an analysis of the number of fulfilled FRs. Therefore, evaluation applying Independence Axiom is conducted when there isn't a single concept that satisfies the set of FRs. This second part of the evaluation starts with an analysis of non-diagonal elements in the design matrix. Non-diagonal elements, i.e. couplings, found in one concept can perhaps be resolved by taking a partial 
solution from another concept which lacks that nondiagonal element.

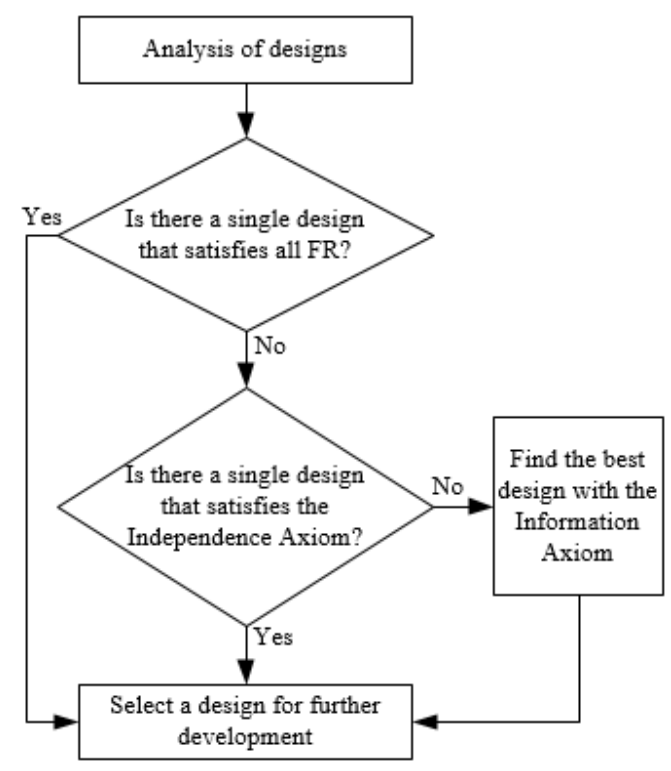

Fig. 2. Modified flowchart of the application of Axiomatic Design

This step is followed by the coupling analysis of the created design matrices. Design matrices of the highest level cannot be solely used for evaluation because they aren't related to concepts, but present general decomposition of the design problem. Therefore, lowerlevel design matrices are integrated into upper-level matrices until the single final matrix for all developed concepts is defined. If a concept does not fulfil a certain FR or lacks a specific DP, corresponding row or column needs to be removed from the design matrix creating a smaller matrix for that concept. Due to possible design complexity, obtained design matrices can be large, which is why reangularity and semiangularity should be calculated to determine the coupling measure for specific concepts. This calculation enables direct comparison of concepts based on satisfaction of the Independence Axiom. However, calculation of semiangularity and reangularity metrics often requires a substantial amount of data and is, therefore, more appropriate for later stages of the design process. This data could be obtained from computer simulations (e.g. FEM and kinematic analysis) or analysis of produced prototypes.

If a final decision cannot be made based on these steps, the Information Axiom is applied, because, unlike the Independence Axiom, it can take into consideration other requirements that have been set on the product. Various constraints can be used to calculate the information content and help choose the best concept. Values of these constraints can be used as the design range (DR), and the ability of the system to fulfil these values is called the system range (SR). The probability of success or, in other words, the information content, is the intersection between the design range and system range. The concept with the smallest information content is the best one according to the Information Axiom.

\section{Application of proposed methodology}

Within the scope of a student product development project course, new mobility scooter concepts were developed. Mobility scooters are electrically powered vehicles designed specifically for the people with limited mobility. The aim of the student development project was to develop a mobility scooter which can be folded and adjusted according to the user needs while considering its aesthetics and ergonomics constraints. At the end of the project, prototypes of developed concepts were produced. This enabled measurement of the fulfilment of the desired requirements set at the beginning of the project. Proposed evaluation methodology was afterwards applied to developed concepts in order to illustrate its application on the student project.

\subsection{Defining requirements}

Evaluation criteria for the case study were derived from customer needs, constraints and non-functional requirements. Customer needs were defined from information gathered through market research and interviews with customers. Most important CNs include the need for a mobility scooter to be lightweight enough for a single person to lift and carry, the possibility of transporting in a car trunk and airport luggage, and the ability to adjust the vehicle so that it suits various people, regardless of their height and mobility. In addition to this, non-functional requirements were defined, because they contain features essential to the customer which couldn't be incorporated into functional requirements (e.g. easy to clean). Industrial partner provided input constraints which present target values for parameters measured on produced prototypes (e.g. mass needs to be under $10 \mathrm{~kg}$ ). Legal obligations are translated into system constraints which must be met (e.g. max speed of $6 \mathrm{~km} / \mathrm{h}$ ).

Functional requirements on the highest level are defined from customer needs regardless of the partial solutions provided in concepts. Satisfying the FRs set on the highest level is a priority because otherwise the basic product functions aren't fulfilled. The defined set of FRs on the highest level is then decomposed to determine FRs on lower levels.

\subsection{Generating design matrices}

Design parameters of each level are obtained through the standard process of zigzagging as explained in Section 2. This zigzagging procedure results in initial design matrices that define relationships between FRs and DPs of each level separately and cannot be used for concept evaluation. The process of determining initial design matrices is iterative to ensure that FRs defined by decomposition are mutually exclusive and collectively exhaustive (MECE) [19]. In a collectively exhaustive decomposition, higher-level FR is completely defined with corresponding lower-level FRs. Mutually exclusive decomposition results in lower-levels FRs that do not 
overlap. As such, this process describes the minimum set of FRs that entirely characterises the design objective [4]. However, developed concepts can contain additional functional requirements that can result in deviations from the ideal design. Without introducing additional FRs, it wouldn't be possible to conduct the detailed evaluation.

Design matrices are filled with symbols $X$ in case of an existing relationship between specific FR and a corresponding DP, and 0 , when there is no relationship between FR and DP. In other words, the designer determines which FRs and DPs are coupled. The relationship can be defined with a design equation to determine the exact value, but it usually assumes the value of 1 if an FR and a DP are completely coupled.

Design matrix of the highest level is upper triangular (decoupled) [Table 1]. Design matrices on lower levels need to be uncoupled or also upper triangular to satisfy the Independence Axiom. In other words, coupling that appeared on the highest level of decomposition must be reflected on lower levels as well. Subsequently, the coupling is affected by changing the order of fulfilling functional requirements. The order of rows in the design matrices indicates the order of fulfilling FRs.

Mobility scooter design was decomposed on 3 levels, and the total of 30 FRs and DPs was defined. For conciseness, only 3 out of 9 initial design matrices will be shown and explained.

Table 1. Decomposition on the highest level

\begin{tabular}{|c|c|c|}
\hline INDEX & FR & DP \\
\hline $\mathbf{1}$ & Ensure load capacity & $\begin{array}{c}\text { Rigidity of basic } \\
\text { structure }\end{array}$ \\
\hline $\mathbf{2}$ & $\begin{array}{c}\text { Allow movement of } \\
\text { parts }\end{array}$ & Movable subsystem \\
\hline $\mathbf{3}$ & $\begin{array}{c}\text { Ensure the flow of } \\
\text { energy required for } \\
\text { movement }\end{array}$ & System for movement \\
\hline $\mathbf{4}$ & $\begin{array}{c}\text { Enable operating of the } \\
\text { vehicle }\end{array}$ & Operating components \\
\hline
\end{tabular}

$$
\left[\begin{array}{l}
F R_{1} \\
F R_{2} \\
F R_{3} \\
F R_{4}
\end{array}\right]=\left[\begin{array}{llll}
X & X & X & O \\
O & X & O & X \\
O & O & X & O \\
O & O & O & X
\end{array}\right]\left[\begin{array}{l}
D P_{1} \\
D P_{2} \\
D P_{3} \\
D P_{4}
\end{array}\right]
$$

By further decomposition of the FR1 Ensure load capacity, another upper triangular matrix was generated [Table 2]. This design matrix for $\mathrm{FR}_{1}$ includes, in addition to others, the symbol $x$, which means that the FR and DP aren't coupled at the moment, but could become in later stages of the design process. We can define that $X>>x$, and the relationship containing $x$ as currently uncoupled, but there is no guarantee of satisfying the Independence Axiom in case the concept is changed. For instance, $\mathrm{DP}_{12}$ Dampening front fork can affect the FR11 Ensure construction rigidity depending on the design.
Table 2. Decomposition of FR $\mathrm{F}_{1}$ Ensure load capacity

\begin{tabular}{|c|c|c|}
\hline INDEX & FR & DP \\
\hline $\mathbf{1 1}$ & $\begin{array}{c}\text { Ensure construction } \\
\text { rigidity }\end{array}$ & Shock-absorbent frame \\
\hline $\mathbf{1 2}$ & $\begin{array}{c}\text { Reduce the shock } \\
\text { impact on the front part } \\
\text { of the construction }\end{array}$ & Dampening front fork \\
\hline $\mathbf{1 3}$ & User positioning & Adjustable seat \\
\hline $\mathbf{1 4}$ & $\begin{array}{c}\text { Secure user against } \\
\text { fallout }\end{array}$ & Safety supports \\
\hline $\mathbf{1 5}$ & $\begin{array}{c}\text { Enable transportation } \\
\text { of additional cargo }\end{array}$ & $\begin{array}{c}\text { Removable storage } \\
\text { space }\end{array}$ \\
\hline
\end{tabular}

$$
\left[\begin{array}{l}
F R_{11} \\
F R_{12} \\
F R_{13} \\
F R_{14} \\
F R_{15}
\end{array}\right]=\left[\begin{array}{lllll}
X & x & O & O & O \\
O & X & O & O & O \\
O & O & X & X & O \\
O & O & O & X & O \\
O & O & O & O & X
\end{array}\right]\left[\begin{array}{l}
D P_{11} \\
D P_{12} \\
D P_{13} \\
D P_{14} \\
D P_{15}
\end{array}\right]
$$

Decomposition of the FR14 Secure user against fallout resulted in decoupled matrix [Table 3]. The DPs on this level are parts of the DP13 Adjustable seat what leads to couplings that potentially have to be resolved. Yet, FRs on the observed level are independent, and the couplings can be shown in the final matrix.

Table 3. Decomposition of FR14 Secure user against fallout

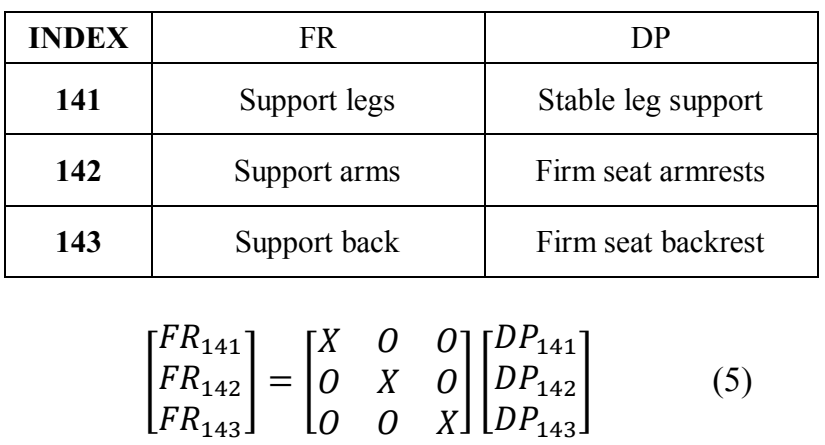

The FR1 Ensure load capacity was decomposed with two matrices shown above. Decomposition is the same for each scooter which makes them comparable.

\subsection{Evaluation based on achieved matrices}

According to the first evaluation step, none of the mobility scooter concepts entirely fulfils the defined set of FRs. Moreover, 3 out of 4 concepts do not allow the transportation of additional cargo (FR15). Other than this FR, concepts only partially fulfil the $\mathrm{FR}_{21}$ Enable folding, meaning that the mobility scooter concepts can be folded but not by satisfying the entire set of FRs on the lower level. This is due to the implementation of FRs that are specific to certain concepts. This problem can occur when evaluating concepts which were not generated using $\mathrm{AD}$ principles since the same higher- 
level FR can be achieved through a different set of lower-level FRs. In such cases, not fulfilling every FR defined on a lower level doesn't imply that a higherlevel FR hasn't been satisfied. Fulfilment of FRs for each concept is shown in a tabular view, to make analysis easier [Table 4]. If a particular concept fulfils an FR, the table contains an $X$, otherwise it contains 0 .

Concept 4 fulfils more FRs in comparison to others and, therefore, its final design matrix contains the highest number of FRs and DPs. However, matrix size doesn't necessarily imply that concept represents a better solution. For instance, every scooter can fulfil FR21 Enable folding and, multiple FRs on its lower decomposition level present a complicated folding procedure for the user. In other words, additional FRs can be perceived as redundant. Since Concept 2 can fulfil all the higher-level FRs (Enable transportation of additional cargo, Enable folding and Adjust vehicle to the user), it is considered as a better concept, even though concept 4 fulfils all lower-level FRs for adjusting the vehicle to the user. Concepts 1 and 3 were identified as weaker solutions based on this first step of evaluation.

Table 4. Evaluation based on fulfilment of FRs that haven't been satisfied in all concepts

\begin{tabular}{|c|c|c|c|c|c|}
\hline Functional & uirements (FR) & $\begin{array}{l}\overline{0} \\
\overline{0} \\
0 \\
\tilde{0}\end{array}$ & $\begin{array}{l}\sim \\
\tilde{z} \\
0 \\
\tilde{0} \\
\tilde{0}\end{array}$ & 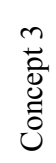 & 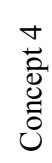 \\
\hline $\begin{array}{l}\text { Enable } t \\
\text { addition }\end{array}$ & $\begin{array}{l}\text { sportation of } \\
\text { argo }\left(\mathrm{FR}_{15}\right)\end{array}$ & 0 & $\mathrm{X}$ & 0 & 0 \\
\hline & $\begin{array}{l}\text { Alter frame } \\
\text { length }\left(\mathrm{FR}_{211}\right)\end{array}$ & $\mathrm{X}$ & 0 & 0 & 0 \\
\hline$\underset{00}{\stackrel{\overrightarrow{2}}{2}}$ & $\begin{array}{l}\text { Rotate seat } \\
\text { carrier }\left(\mathrm{FR}_{212}\right)\end{array}$ & $\mathrm{X}$ & 0 & $\mathrm{X}$ & $X$ \\
\hline $\begin{array}{l}\frac{5}{0} \\
\frac{0}{0} \\
\frac{0}{0} \\
\frac{0}{\sigma}\end{array}$ & $\begin{array}{c}\text { Enable tiller } \\
\text { folding } \\
\left(\mathrm{FR}_{213}\right)\end{array}$ & $\mathrm{X}$ & 0 & 0 & $X$ \\
\hline 武 & $\begin{array}{c}\text { Enable frame } \\
\text { folding } \\
\left(\mathrm{FR}_{214}\right)\end{array}$ & 0 & $\mathrm{X}$ & X & $X$ \\
\hline 芯 & $\begin{array}{l}\text { Adjust tiller } \\
\text { height }\left(\mathrm{FR}_{231}\right)\end{array}$ & 0 & $\mathrm{X}$ & 0 & $\mathrm{X}$ \\
\hline 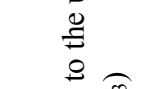 & $\begin{array}{l}\text { Adjust tiller } \\
\text { angle }\left(\mathrm{FR}_{232}\right)\end{array}$ & 0 & 0 & 0 & $X$ \\
\hline 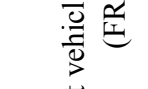 & $\begin{array}{c}\text { Adjust seat } \\
\text { height ( }\left(\mathrm{FR}_{233}\right)\end{array}$ & 0 & $\mathrm{X}$ & 0 & $\mathrm{X}$ \\
\hline 莺 & $\begin{array}{c}\text { Enable seat } \\
\text { rotation } \\
\left(\mathrm{FR}_{234}\right)\end{array}$ & 0 & $\mathrm{X}$ & 0 & $\mathrm{X}$ \\
\hline
\end{tabular}

The next step in evaluation is the analysis of nondiagonal elements in the final matrices of each concept. Table 5 shows how many non-diagonal elements are shared between different concept solutions. This comparison may indicate which independencies designers most often fail to satisfy. In addition, it can support designers in their search for partial solutions in other concepts that managed to satisfy Independence Axiom.

Table 5. Number of common and total number of non-diagonal elements in concepts

\begin{tabular}{|c|c|c|c|c|}
\hline & $\begin{array}{c}\text { Concept } \\
1\end{array}$ & $\begin{array}{c}\text { Concept } \\
2\end{array}$ & $\begin{array}{c}\text { Concept } \\
3\end{array}$ & $\begin{array}{c}\text { Concept } \\
4\end{array}$ \\
\hline $\begin{array}{c}\text { Concept } \\
1\end{array}$ & 20 & 21 & 22 \\
\hline $\begin{array}{c}\text { Concept } \\
2\end{array}$ & 20 & 21 & 21 & 29 \\
\hline $\begin{array}{c}\text { Concept } \\
3\end{array}$ & 21 & 29 & 22 & 22 \\
\hline $\begin{array}{c}\text { Concept } \\
4\end{array}$ & 22 & $\mathbf{3 6}$ & $\mathbf{2 2}$ & $\mathbf{4 0}$ \\
\hline $\begin{array}{c}\text { Total no. } \\
\text { of non- } \\
\text { diagonal } \\
\text { elements }\end{array}$ & $\mathbf{2 4}$ & & & \\
\hline
\end{tabular}

The total number of non-diagonal elements represents how many couplings appear in the final design matrix of each concept. Concept 4 has the highest total number of non-diagonal elements. This is partially due to the many FRs defined for folding of the scooter and adjusting it to the user. Concept 3 has the lowest number of FRs for folding and adjusting the vehicle, and the smallest number of non-diagonal elements. Comparison of the total number of non-diagonal elements and the common number of elements can sometimes indicate a common issue among different designs. In this case study, there are 19 non-diagonal elements common to all four concepts. Due to such a high number of non-diagonal elements, it can be concluded that maintaining the independence of FR21 Enable folding and FR23 Adjust vehicle to the user was a difficult task for designers. Beside these two FRs, by analysing the location of non-diagonal elements, partial solutions for couplings can be found and applied to other concepts. For instance, DP311 Li-ion battery is dependent on the FR46 Usable in darkness. This coupling can be resolved by providing an independent power source for the light that every scooter must have due to legal obligations.

Afterwards, concept comparison was done by calculating reangularity and semiangularity with equations given in Section 2 of this paper. At this stage of the design process, there was not enough information to determine the exact coupling values. Therefore, design matrices were populated with values based on designers' perception of coupling severity in the following way: $X$ equals $1, x$ equals 0,1 due to the condition $X>>x$, and 0 remained 0 . Calculated values are shown in [Table 6].

Table 6. Concept comparison by reangularity and semiangularity values

Reangularity Semiangularity 


\begin{tabular}{|l|l|l|}
\hline Concept 1 & 0,3266 & 0,001889 \\
\hline Concept 2 & 0,3952 & 0,000564 \\
\hline Concept 3 & 0,3462 & 0,003646 \\
\hline Concept 4 & 0,2933 & 0,000344 \\
\hline
\end{tabular}

These values imply that none of the concepts satisfies the Independence Axiom. To our knowledge, literature doesn't provide guidelines whether the concept for further development should be the one with the highest value of reangularity ( $\mathrm{R}=1$ for uncoupled designs) or the one with the smallest difference between reangularity and semiangularity $(\mathrm{R}=\mathrm{S}$ for a decoupled design). Concept 2 and Concept 4 are closer to satisfying the desired design matrix than concepts 1 and 3, but are still far away from fulfilling the Independence Axiom. Therefore, a final decision wasn't made and, the Information Axiom had to be applied for further evaluation.

In this final step, input constraints provided by the industrial partner were compared to the values measured on the prototypes [Table 7]. In addition to this, folding time was added as an important aspect for the users. The information content is calculated with equations provided in [10] where the SR is achieved value on a concept, and the $\mathrm{CR}$ is input constraint value. Information content is defined as zero if the achieved value is smaller or the same as required.

Table 7. Concept comparison based on Information Axiom

\begin{tabular}{|c|c|c|c|c|c|}
\hline Criteria & 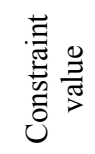 & 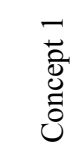 & $\begin{array}{l}N \\
\stackrel{0}{0} \\
0 \\
0 \\
0\end{array}$ & 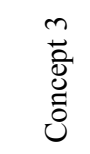 & 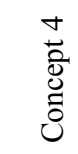 \\
\hline Mass [kg] & $\leq 10$ & 26,1 & 23,1 & 26,3 & 24,5 \\
\hline $\begin{array}{l}\text { Folding } \\
\text { time }[\mathrm{s}]\end{array}$ & $\begin{array}{c}\min = \\
13\end{array}$ & 20 & 13 & 27 & 40 \\
\hline $\begin{array}{l}\text { Length } \\
{[\mathrm{mm}]}\end{array}$ & $\leq 900$ & 950 & 810 & 950 & 780 \\
\hline $\begin{array}{l}\text { Height } \\
{[\mathrm{mm}]}\end{array}$ & $\leq 700$ & 770 & 598 & 700 & 650 \\
\hline Width [mm] & $\leq 400$ & 620 & 670 & 550 & 870 \\
\hline $\begin{array}{c}\text { Total } \\
\text { information } \\
\text { content }\end{array}$ & - & 2,853 & 1,952 & 2,987 & 4,035 \\
\hline
\end{tabular}

Since the ideal information content is 0 , it can be concluded that Concept 2 is the best solution according to the Information Axiom. It has the smallest mass and the least folding time which are two aspects of the utmost importance for the user. On the other hand, Concept 4 which fulfils more FRs has proven to be more complicated compared to other scooters and particularly, Concept 2.
This application represents four steps explained in the proposed modified methodology for the concept evaluation with $\mathrm{AD}$ principles. Each step provided different insights into the strengths and weaknesses of the analysed concepts. The application also showed that results are inconsistent by using different $\mathrm{AD}$ evaluation criteria. Using the Independence Axiom, Concept 4 is perceived as the best solution due to the least amount of changes that are required for satisfaction and fulfilment of FRs. However, other steps in the evaluation, like calculation of reangularity and semiangularity values, non-diagonal elements and the Information Axiom, raised serious concerns about Concept 4 due to additional FRs. These results may be a consequence of working with concepts that weren't generated using $\mathrm{AD}$ principles since it can be hard to retroactively achieve the minimum set of FRs like AD principles demand. If the evaluation was based solely on the Independence Axiom, as is usually the case, Concept 4 could've been chosen as the best one. This application shows that a more extensive validation of various concept evaluation steps should be taken in further studies to embrace different criteria and aspects of the proposed solutions.

\section{Discussion}

Application of $\mathrm{AD}$ principles to concept evaluation relies more on technical knowledge, compared to other methods used for evaluation, since the designer reformulates the needs perceived by the customer into the functional requirements [12]. Therefore, this results in an evaluation process based on the fulfilment and independence of functional requirements and may reduce biased personal judgments [7]. However, Axiomatic Design doesn't provide a way to check whether the CNs have been adequately reformulated to FRs. In addition, there is still no detailed procedure for defining FRs. Other than defining FRs, it is crucial to specify nontechnical requirements and constraints properly. Until now, these other types of information usually haven't been incorporated into the evaluation process.

In addition, literature doesn't acknowledge the difference of evaluating concepts generated with or without AD principles. Concepts that weren't generated with $\mathrm{AD}$ principles aren't improved to satisfy the Independence Axiom within the scope of the evaluation. However, conducted evaluation suggests improvements which can be applied in the later stages of the design process.

The proposed methodology suggests an implementation of constraints for the design range as a part of the Information Axiom. The implementation can be hard to conduct if there is not enough information about target values for concepts. However, implemented constraints can show to what extent the customer needs are satisfied. Examples found in literature mostly compared a single concept to its ideal solution [10]-[15]. Previously, concepts were often not compared to one another due to their different decompositions on lower levels. The proposed methodology aims to avoid this problem by generating a general common matrix for 
each concept that includes FRs found in all of them. If the concepts aren't comparable in such way, evaluation cannot be done based on fulfilment of FRs and number of total and shared non-diagonal elements. On the other hand, if the evaluation based on fulfilment of FRs isn't conducted, an incomplete solution could be chosen.

One of the advantages of applying the proposed methodology is the ability to combine different solutions from various concepts to solve certain couplings. Analysis of non-diagonal elements provides insight about couplings that are the most difficult for the designer to resolve. It also enables easy comparison of different solutions which can be beneficial when combining different concepts. These first two steps, the fulfilment of FRs and analysis of non-diagonal elements, are suitable for application in the earlier phases of conceptualisation. In addition, they are easy to implement.

In this case study, application of Independence Axiom in terms of calculating coupling measures (reangularity and semiangularity) hasn't provided sufficient information for concept selection. This may be due to the small differences between the obtained concept values and inadequate definition of the coupling values. Therefore, the Information Axiom was applied to the concepts that didn't satisfy the Independence Axiom, even though Axiomatic Design theory advises against it. Unlike the first two steps, calculating the coupling measures and the Information Axiom usually requires more data and is often more appropriate to implement in later stages of the design process. More case studies will be conducted to provide additional validation and testing of the proposed methodology.

\section{Conclusion}

The modified methodology proposed in this paper aims to solve reoccurring issues found in the literature about various aspects of concept evaluation using AD principles. The underlying logic of the proposed methodology suggests a definition of the common matrices for developed concepts and includes four subsequent steps to enable proper concept evaluation. Each of the evaluation steps showed its advantages and potential issues as pointed out in the previous section.

The validation of the proposed methodology was carried out by analysing concepts generated during mobile scooter development. After initial matrices were defined, couplings were affected by reformulating FRs and DPs and changing their order in the design matrix. The proposed evaluation methodology showed that none of the concepts fulfils the whole set of FRs and the Independence Axiom. Therefore, the evaluation at this stage was inconclusive, indicating the need for the Information Axiom.

Further research should examine the problem of carrying out different FRs throughout the different phases of the product lifecycle. For instance, the user either adjusts the mobility scooter or unfolds/folds it, but never simultaneously. Currently, such cases are usually presented as coupling even though it is preferable to have the same product fulfil different functions at different times. Coupling measures, $\mathrm{R}$ and $\mathrm{S}$, should be also further studied to ensure the proper analysis and interpretation of obtained values. Although there are many ways to apply the Information Axiom, further research should be conducted to enable implementation of different types of requirements and criteria.

The case project was a part of the Erasmus+ Strategic Partnership programme and funded with support from the European Commission. This communication reflects the views of the authors, and the Commission cannot be held responsible for any use which may be made of the information contained therein.

\section{References}

[1] K. T. Ulrich and S. D. Eppinger, Product Design and Development, 5th ed. 2012.

[2] D. G. Ullman, The Mechanical Design Process. New York: McGraw-Hill, 2010.

[3] G. Pahl, W. Beitz, J. Feldhusen, and K.-H. Grote, Engineering Design, 3rd ed. SpringerVerlag, 2007.

[4] N. P. Suh, The Principles of Design. Oxford University Press, 1990.

[5] Y. Borgianni and D. T. Matt, "Ideality in Axiomatic Design and beyond," in Procedia CIRP, 2016, vol. 53, pp. 95-100.

[6] N. P. Suh, Complexity: Theory and Application. Oxford University Press, 2005.

[7] A. Xiao, S. S. Park, and T. Freiheit, “A Comparison of Concept Selection in Concept Scoring and Axiomatic Design Methods," Proc. Can. Eng. Educ. Assoc., 2011.

[8] O. Kulak, S. Cebi, and C. Kahraman, "Applications of axiomatic design principles: A literature review," Expert Syst. Appl., vol. 37, no. 9, pp. 6705-6717, 2010.

[9] A. Kreuzer, B. Nitsche, and J. Kantola, "Application of Axiomatic Design to Electric Bicycles," Proc. ICAD2014, Eight Int. Conf. Axiomat. Des., pp. 35-42, 2014.

[10] B. El-Haik, Axiomatic Quality: Integrating Axiomatic Design with Six-Sigma, Reliability, and Quality Engineering. John Wiley \& Sons, 2005.

[11] G.-J. Park, Analytic Methods for Design Practice. Springer Science \& Business Media, 2007.

[12] D. Silverstein, P. Samuel, and N. Decarlo, Axiomatic Design, vol. 1. 2011.

[13] G. Heo and S. K. Lee, "Design evaluation of emergency core cooling systems using Axiomatic Design," Nucl. Eng. Des., vol. 237, no. 1, pp. 38-46, 2007. 
[14] J. H. Kim, K. S. Kim, and Y. J. Kang, "Ride comfort evaluation and suspension design using axiomatic design," J. Mech. Sci. Technol., vol. 21, no. 7, pp. 1066-1076, 2007.

[15] A. Girgenti, A. Giorgetti, C. Monti, and P. Citti, "Conceptual Design Evaluation of Different Friction Devices Through the Use of Axiomatic Design," Proc. ICAD2014, Eighth Int. Conf. Axiomat. Des., 2014.

[16] J. Qiao and J. Shang, "Application of axiomatic design method in in-pipe robot design," Robot. Comput. Integr. Manuf., vol. 29, no. 4, pp. 4957, 2013.

[17] G. Bragason, S. Porsteinsson, R. I. Karlsson, N. Grosse, and J. T. Foley, "Heat-activated Parachute Release System," Proc. 9th Int. Conf. Axiomat. Des., vol. 34, pp. 131-136, 2015.

[18] M. K. Thompson, "Where Is the 'Why' in Axiomatic Design?," Proc. ICAD2014 Eighth Int. Conf. Axiomat. Des., pp. 7-12, 2014.

[19] C. A. Brown, "Decomposition and prioritization in engineering design," Proc. 6th Int. Conf. Axiomat. Des., pp. 41-47, 2011. 